\title{
Hemodynamic Features in Stenosed Coronary Arteries: CFD Analysis Based on Histological Images
}

\author{
Mahsa Dabagh,, ${ }^{1,2}$ Wakako Takabe, ${ }^{2,3}$ Payman Jalali, ${ }^{1}$ Stephan White, ${ }^{4}$ and Hanjoong Jo ${ }^{2,3}$ \\ ${ }^{1}$ LUT School of Technology, Lappeenranta University of Technology, 53851 Lappeenranta, Finland \\ ${ }^{2}$ Department of Cardiology, Emory University, Atlanta, GA 30322, USA \\ ${ }^{3}$ Department of Biomedical Engineering, Georgia Institute of Technology, Atlanta, GA 30332, USA \\ ${ }^{4}$ Faculty of Medicine and Density, University of Bristol, Bristol BS8 1TH, UK
}

Correspondence should be addressed to Mahsa Dabagh; mahsa@lut.fi

Received 8 March 2013; Revised 30 May 2013; Accepted 30 May 2013

Academic Editor: Chang-Hwan Im

Copyright (C) 2013 Mahsa Dabagh et al. This is an open access article distributed under the Creative Commons Attribution License, which permits unrestricted use, distribution, and reproduction in any medium, provided the original work is properly cited.

\begin{abstract}
Histological images from the longitudinal section of four diseased coronary arteries were used, for the first time, to study the pulsatile blood flow distribution within the lumen of the arteries by means of computational fluid dynamics (CFD). Results indicate a strong dependence of the hemodynamics on the morphology of atherosclerotic lesion. Distinctive flow patterns appear in different stenosed regions corresponding to the specific geometry of any artery. Results show that the stenosis affects the wall shear stress (WSS) locally along the diseased arterial wall as well as other adjacent walls. The maximum magnitude of WSS is observed in the throat of stenosis. Moreover, high oscillatory shear index (OSI) is observed along the stenosed wall and the high curvature regions. The present study is capable of providing information on the shear environment in the longitudinal section of the diseased coronary arteries, based on the models created from histological images. The computational method may be used as an effective way to predict plaque forming regions in healthy arterial walls.
\end{abstract}

\section{Introduction}

Atherosclerosis in the coronary arteries tends to be localized in regions with curvature and branching associated with complex, unsteady, and turbulent pattern of the flow [1]. In these regions, the fluid shear stress deviates from its normal spatial and temporal distribution patterns in straight vessels. The role of WSS in the localization of the atherosclerosis has been widely accepted [1-6]. Shahcheraghi et al. [3] explained clinically the fact that a low shear stress region is generally found in the downstream of developing stenotic plaques, leading to an increased density of leaky junctions associated with an increased level of low-density-lipoprotein (LDL) flux into the arterial wall. Moreover, the OSI is known as the predictor of formation of atherosclerosis and vulnerability for plaque in coronary arteries [7-9]. High OSI has been widely used as indicators of atherosclerosis because the regions with high OSI are likely to experience stagnation or backflow [711]. This is strongly related to the progression of atherosclerosis because it affects the arrangement of endothelial cells in adjacent tissues. It has been shown that the areas with high values of OSI are usually located in the regions where wall shear stress is low [7]. Taking all together, the creation and development of atherosclerosis depend not only on the biological features but also on the biomechanical factors, including WSS and the cyclic force caused by the pulsatile blood pressure. Therefore, a full description of the flow pattern through the diseased coronaries, with the emphasis on the patient-specific data, is essential for quantifying the wall shear stress that may occur in a stenosed artery during the cardiac cycle. The knowledge will also lead to find the most vulnerable sites for the plaque rupture.

Previous studies have shown the flow pattern through the idealized geometry [6-8] or the reconstructed geometry of 


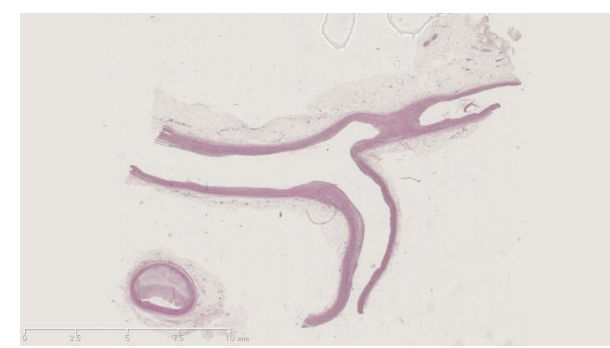

(a)

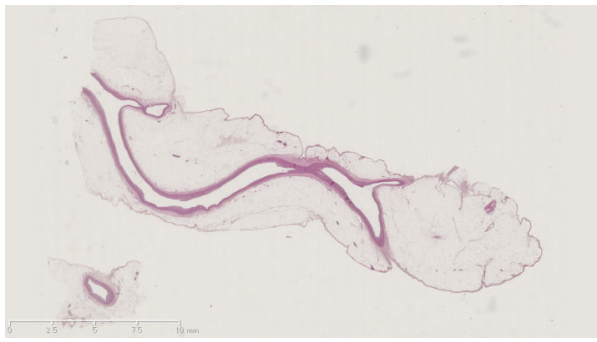

(c)

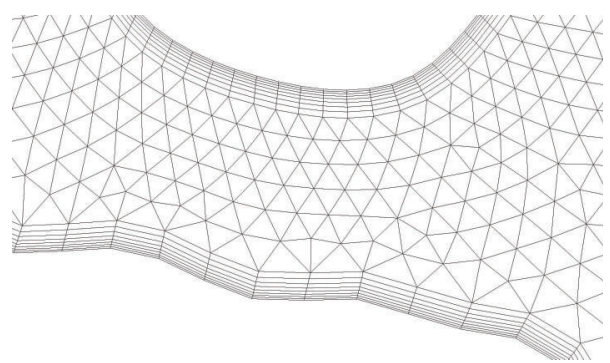

(e)

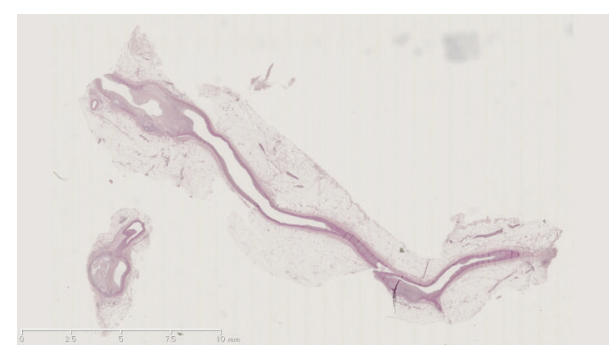

(b)

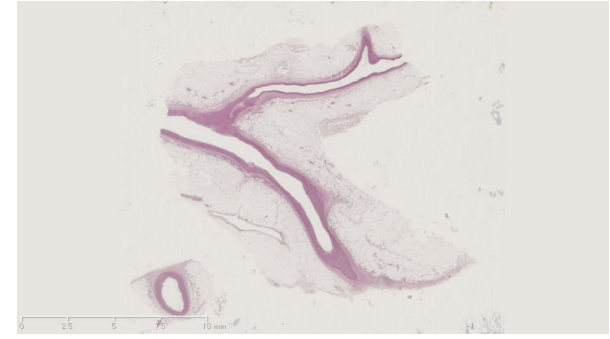

(d)

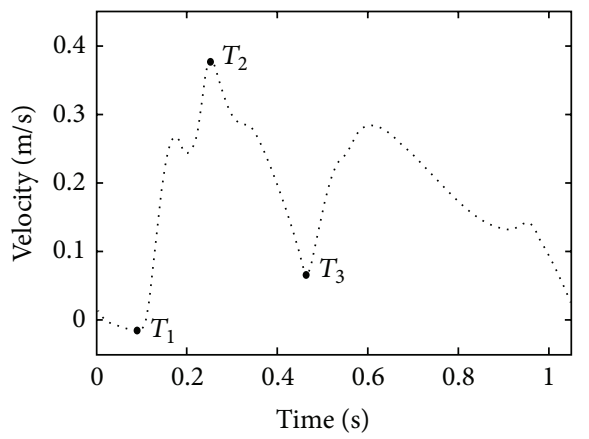

(f)

FIGURE 1: (a)-(d) LS images of four diseased coronary arteries, (e) the computational meshes at the stenosis location, and (f) velocity pulse at the inlet.

arteries from a series of slices obtained using computerized tomography (CT) scan images $[9,10]$, magnetic resonance images (MRI) [11], the spiral CT scan [12], the phase-contrast MRI method [13], or intravascular ultrasound (IVUS) [4]. However, simplifications were applied in these studies; for example, the cross-section of the artery was approximated as circles which fit the centerline to a cubic spline curve $[7,13]$, or a constant diameter was applied throughout the model $[13,14]$.

In the present study, the histological images from longitudinal section of four diseased coronary arteries were used, for the first time, to investigate the local hemodynamic more precisely through the stenosed artery models. The geometry is reconstructed directly by graphical reading of points on the luminal surface of the arteries. Any simplifications have been avoided in the construction of the model. The pulsatile blood flow pattern was applied through the reconstructed stenosed coronary arteries, and the blood flow characteristics along the arterial wall was analyzed by means of computational fluid dynamics (CFD).

\section{Methods}

2.1. Reconstruction of 2D Geometry of Coronary Artery from Histological Image. Two-dimensional (2D) geometries of the human coronaries are reconstructed from the histological images which were acquired from coronary arteries of four donor hearts (Faculty of Medicine and Density, University of Bristol, Bristol, UK). The samples contained regions with mild to severe atherosclerosis. The diseased coronary arteries were cut in longitudinal section, stained with H\&E, and imaged on a nanozoomer, namely, NanoZoomer 2.0HT (Hamamatsu photonics, Hamamatsu, Japan) with 40x magnification at the pathology core lab in the Winship Cancer Institute, Emory University.

To construct the coronary artery geometries, all the images were processed individually. The images were converted first to the boundary plots within MATLAB v. 7.11.0 (R2010b). The lumen area was identified via image segmentation. Points representing the surface of the endothelium were obtained as the final output of this step. The vertices 


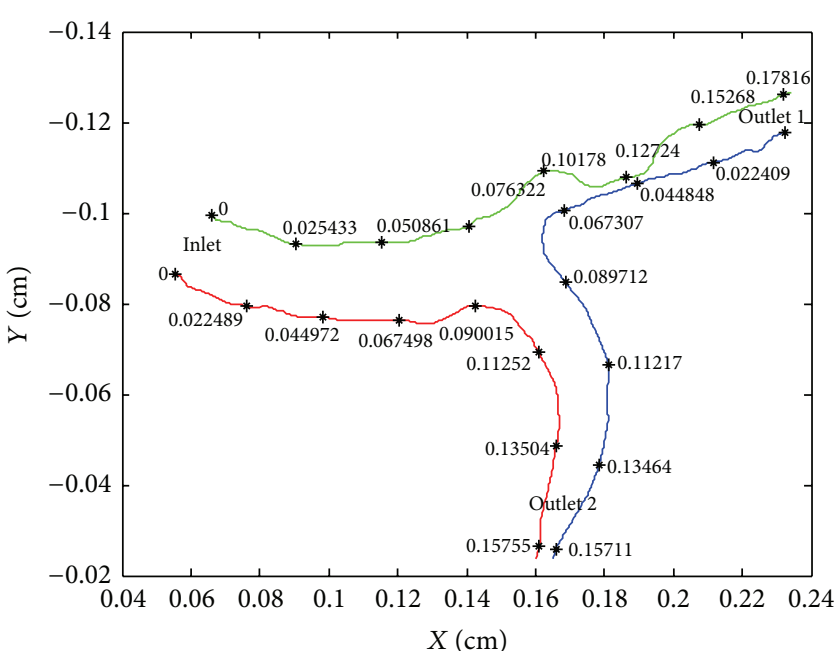

(a)

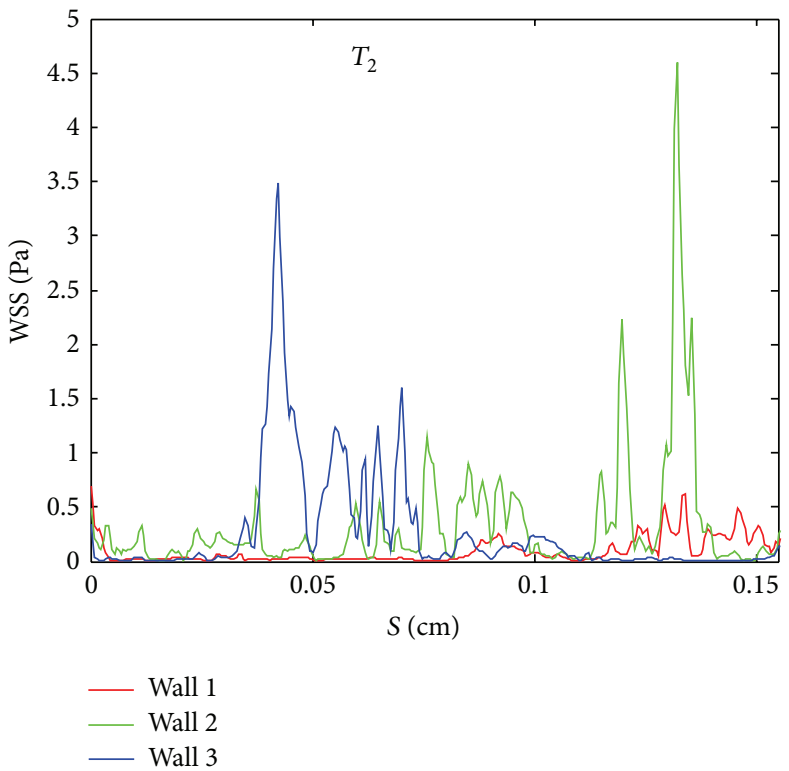

(c)

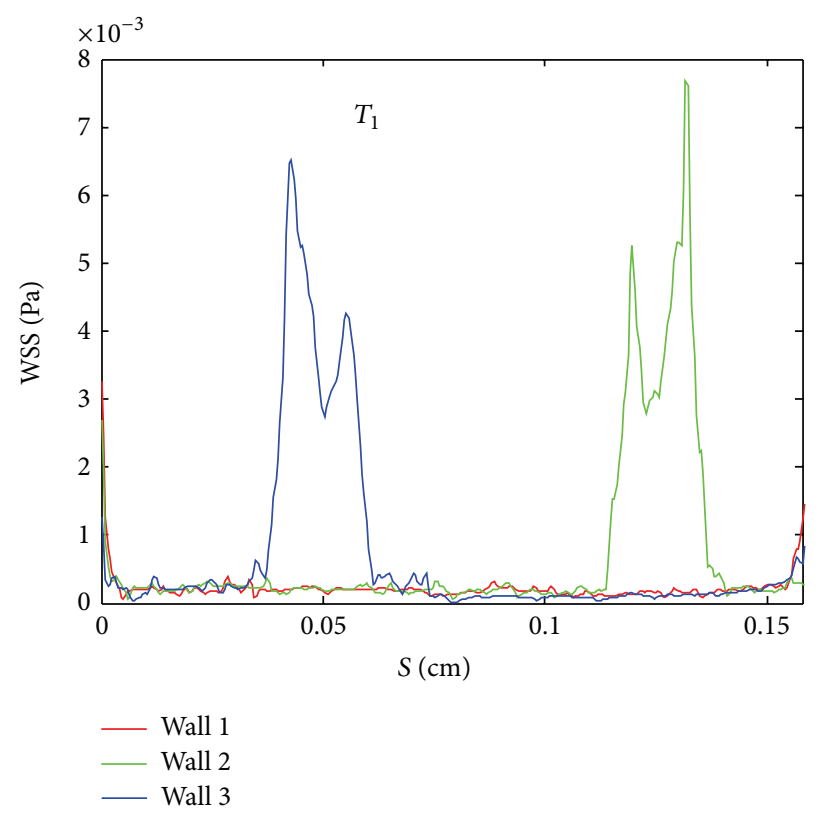

(b)

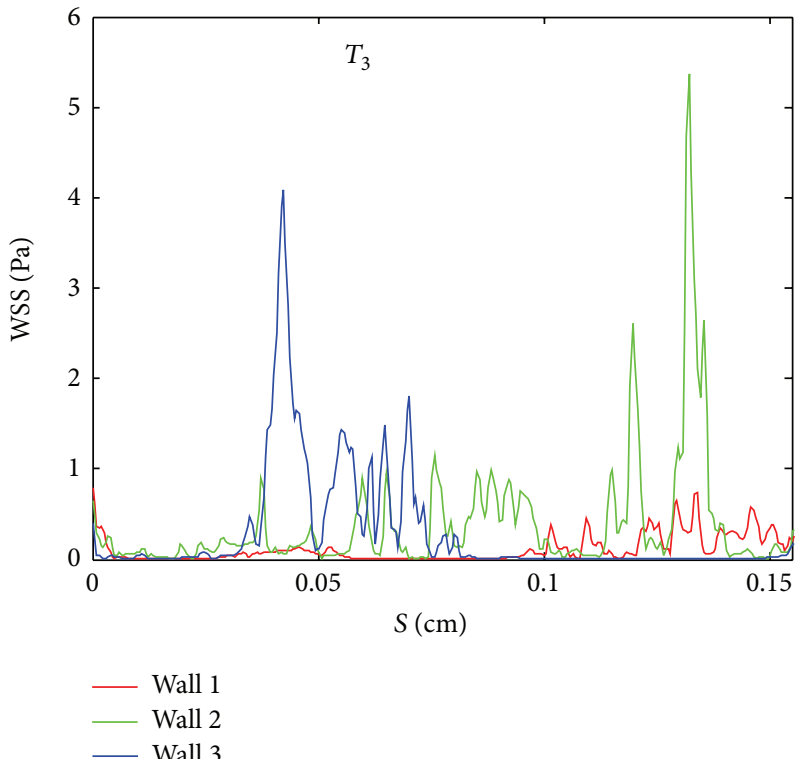

(d)

FIGURE 2: (a) Boundary plots of the coronary arterial walls 1, 2, and 3 shown in red, green, and blue, respectively. (b)-(d) WSS distribution along coronary walls at time $T_{1}, T_{2}$, and $T_{3}$, respectively.

acquired from the processing of the images were connected to generate a $2 \mathrm{D}$ surface representing the lumen of coronary. The 2D model was trimmed with the CFD preprocessing and meshing code Gambit V2.4.6 to generate the flat inlet and outlet boundaries. The computational mesh grid consisted of about 50,000 grid elements. Thin boundary layers were used for the precise calculations near the walls. Several steadystate simulations were performed with different grid sizes to ensure the mesh independence of the results. The boundary layer mesh used in these simulations consists of 6 layers with the first layer attached to the luminal surface with the thickness of $0.01 \mathrm{~mm}$. The thickness of consecutive layers grows with the factor of 1.2. Figures 1(a)-1(d) demonstrate the original histological image of each coronary artery. Figure 1(e) shows the grids generated for the models.

2.2. Governing Equations and Boundary Conditions. The blood flow is assumed to beincompressible with uniform properties including the viscosity of $3.5 \times 10^{-3} \mathrm{~Pa} \cdot \mathrm{s}$ (Newtonian fluid) and the density of $1067 \mathrm{kgm}^{-3}$. The assumption of Newtonian fluid for the blood is valid wherever the shear 


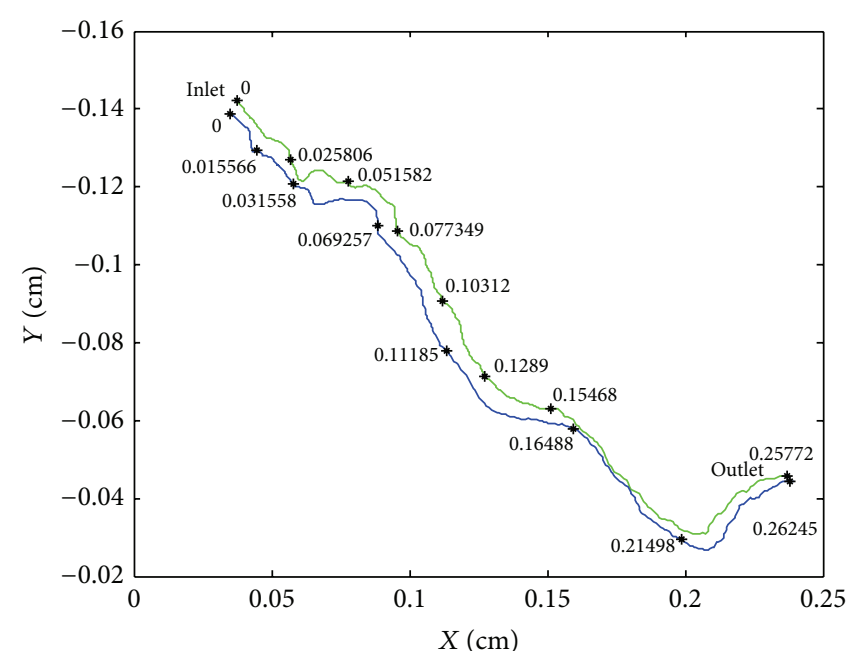

(a)

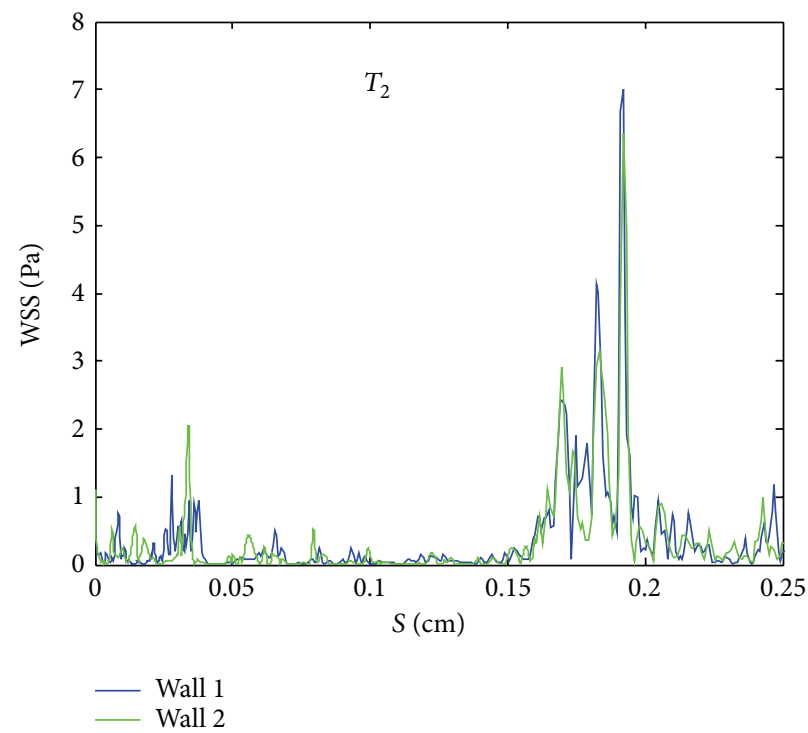

(c)

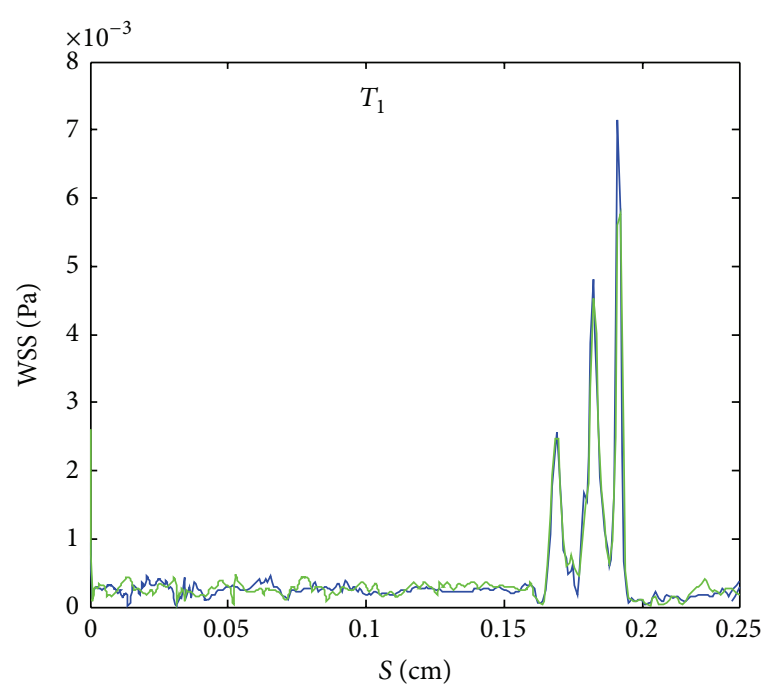

- Wall 1

Wall 2

(b)

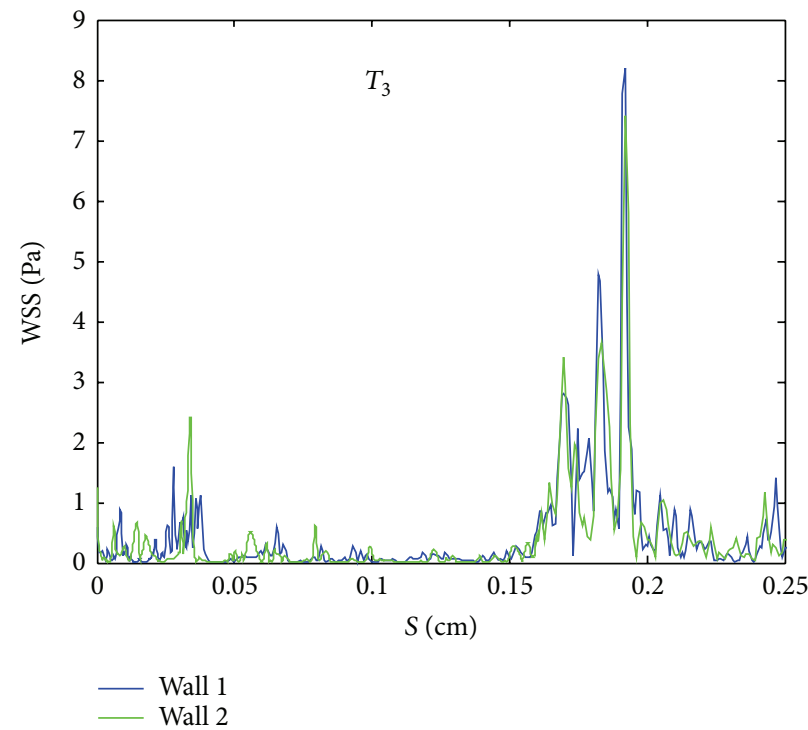

(d)

FIGURE 3: (a) Boundary plots of the coronary arterial walls 1 and 2 shown in green and blue, respectively. (b)-(d) WSS distribution along coronary walls at time $T_{1}, T_{2}$, and $T_{3}$, respectively.

rate is greater than $100 \mathrm{~S}^{-1}[9,10,12,13]$. This assumption is feasible for coronary artery. The time-dependent NavierStokes equations govern the blood flow in the coronary artery, as

$$
\begin{gathered}
\nabla \cdot \vec{U}=0, \\
\rho \frac{\partial \vec{U}}{\partial t}+\rho(\vec{U} \cdot \nabla) \vec{U}=-\nabla p+\mu \nabla^{2} \vec{U},
\end{gathered}
$$

where $\vec{U}$ is the velocity vector, while $p, \rho$, and $\mu$ denote pressure, density, and viscosity, respectively. The maximum Reynolds number, $\operatorname{Re}=\rho \bar{U} D / \mu$ (where $D$ is the inlet diameter of coronary and $\bar{U}$ is the maximum pulsating inflow velocity), is calculated as 232 . The inlet diameter of the distal left anterior descending coronary artery is $1.9 \pm 0.4 \mathrm{~mm}$, and the maximum pulsating inflow velocity is taken as $0.4 \mathrm{~m} / \mathrm{s}$ [14]. Figure 1(e) demonstrates the pulse for inlet velocity. The critical Reynolds number for an unsteady flow ranges from 5000 to 20,000 [15-17]. Therefore, the laminar flow assumption is reasonable in the present simulation.

The pulse of the inlet velocity (Figure $1(\mathrm{f})$ ) applied in the proximal site of the right coronary artery (RCA) is decomposed into a Fourier series of trigonometric basis functions with 3 harmonics. The input velocity shown in Figure 1(f) is based on the velocity waveforms measured with a commercial ultrasound Doppler probe FloWire (Volcano 


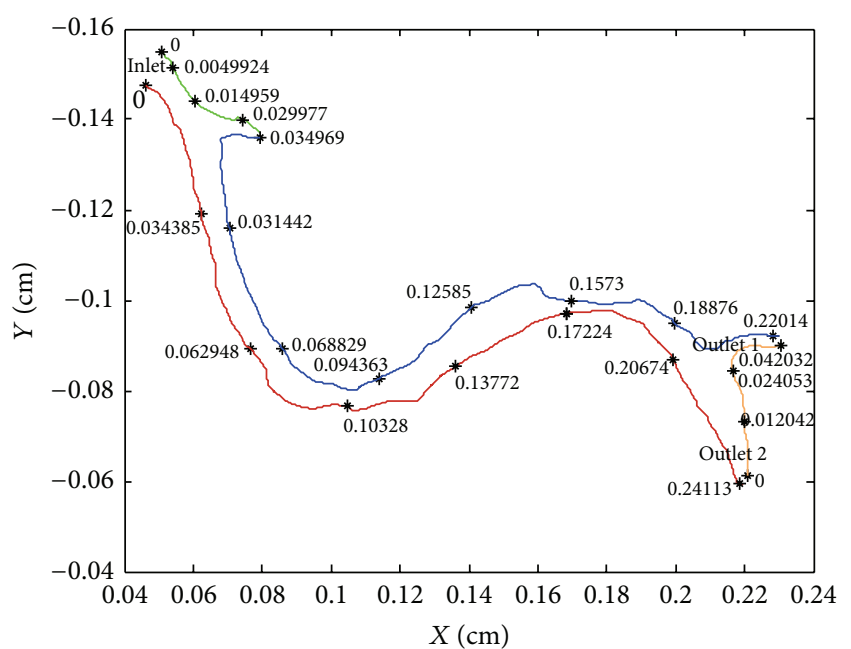

(a)

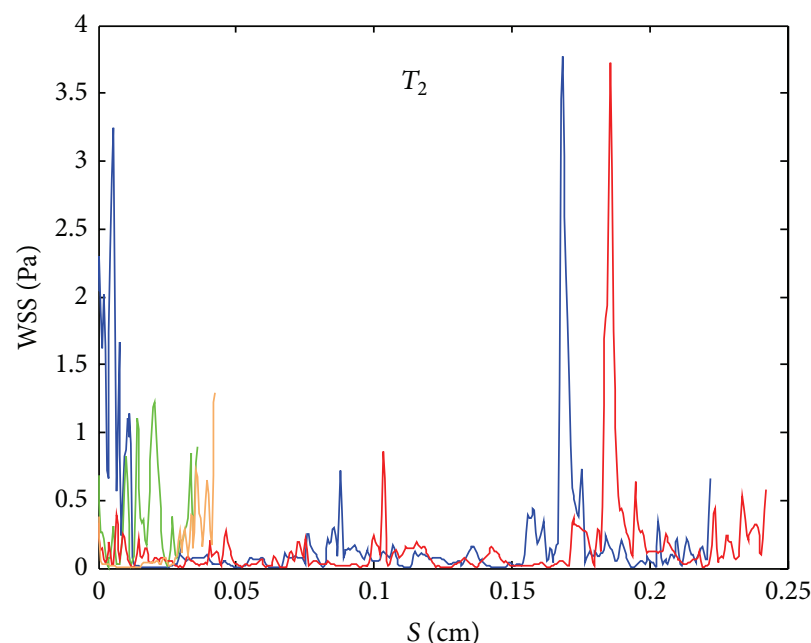

$\begin{array}{ll}\text { Wall } 1 & \text { Wall } 3 \\ \text { Wall } 2 & \text { Wall } 4\end{array}$

(c)

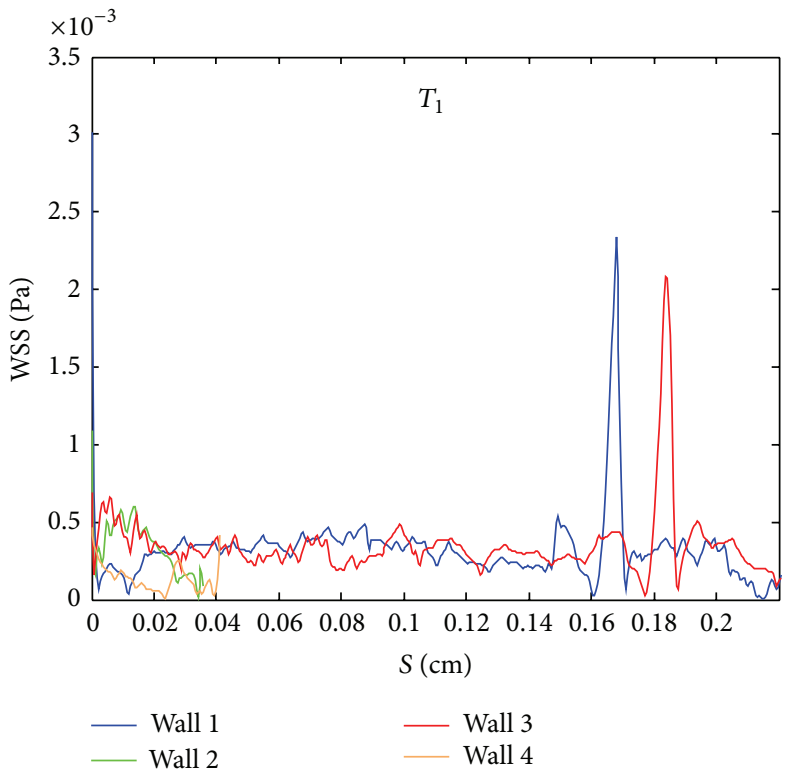

(b)

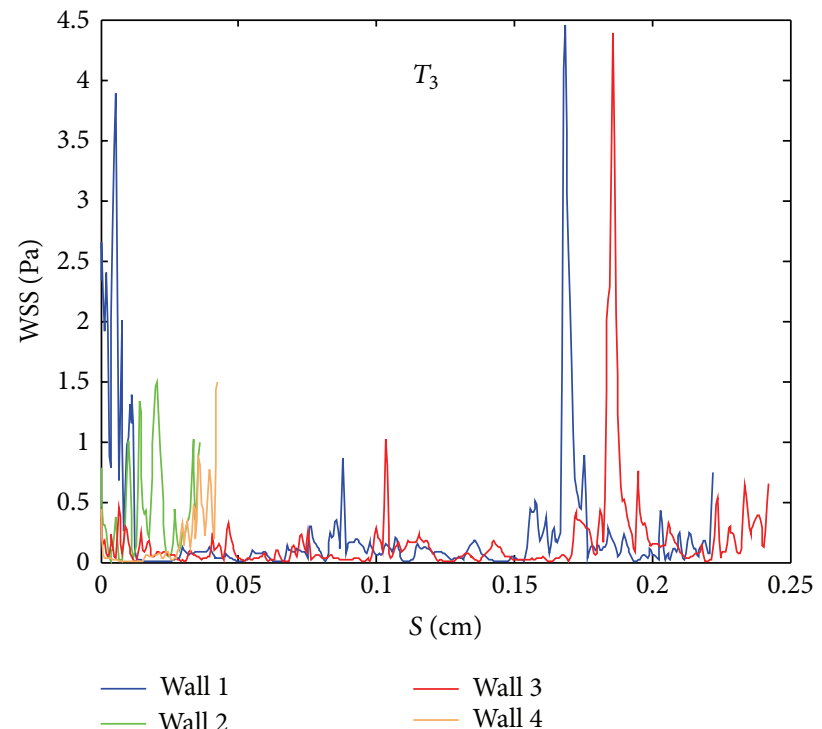

(d)

FIGURE 4: (a) Boundary plots of the coronary arterial walls 1, 2, 3, and 4 shown in red, green, blue, and brown, respectively. (b)-(d) WSS distribution along coronary walls at time $T_{1}, T_{2}$, and $T_{3}$, respectively.

TM Corporation) with $1 \mathrm{kHz}$ sampling rate at the proximal site of RCA, provided by Torii et al. [18]. The inlet velocity was kept constant across the inlet surface at each time instant of the cardiac cycle. The points indicated by $T_{1}$, $T_{2}$, and $T_{3}$ in Figure 1(f) correspond to the diastole, the peak systole, and accelerating phase within the cardiac cycle, respectively. The outflow boundary conditions were imposed at the outlets. The coronary arterial walls are assumed to be rigid with no-slip boundary condition on the luminal surface. The governing equations along with the given boundary conditions are solved with the finite-volume (FV) solver package, Fluent V12.1.4. The SIMPLE algorithm is used for the coupling of the pressure-velocity terms. The simulations are carried out for four inlet cycles to ensure that the spatial and temporal flow dynamics are periodic. The total time of $1 \mathrm{~s}$ with the time step size of $1 \mathrm{~ms}$ is taken for each cycle. The simulations are performed for four cardiac cycles, of which only the results of the forth cycle are demonstrated in the following section at different times to ensure that all the phenomena associated with the initial conditions are damped 


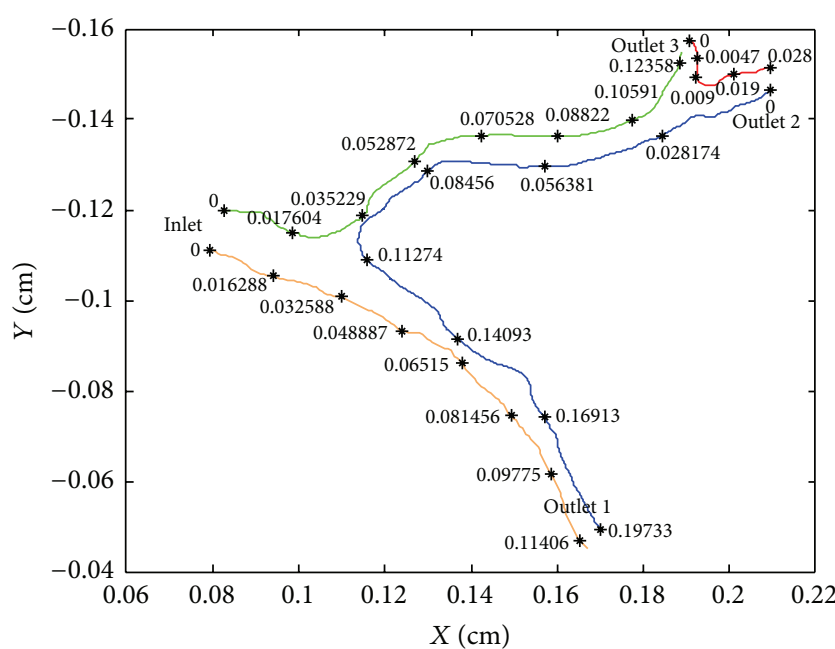

(a)

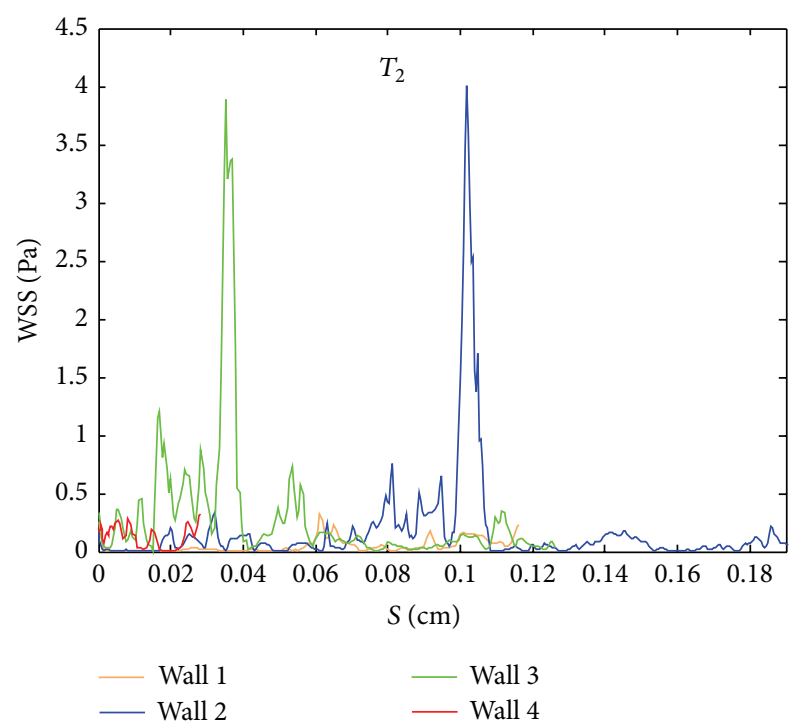

(c)

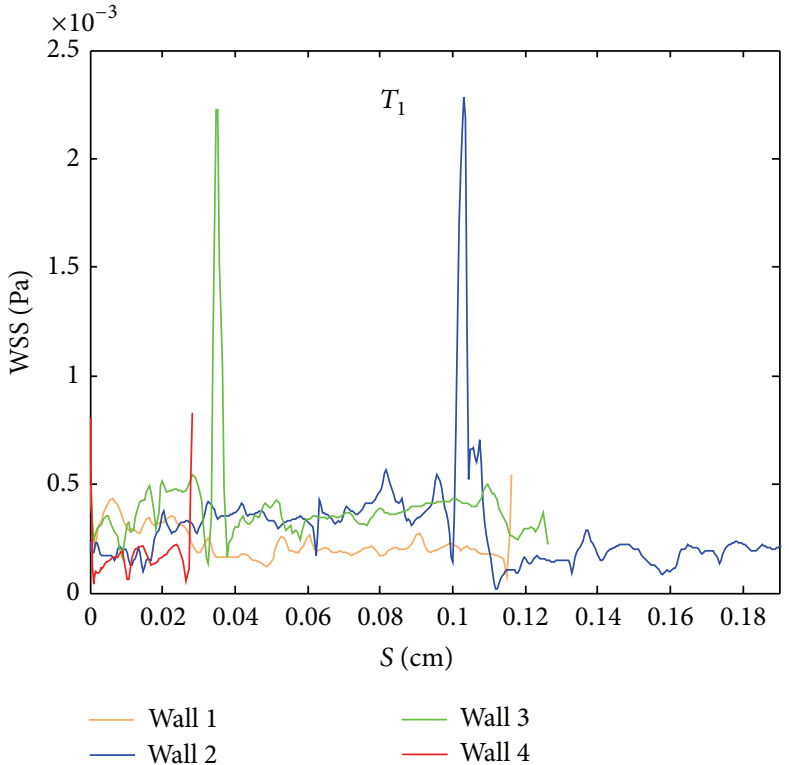

(b)

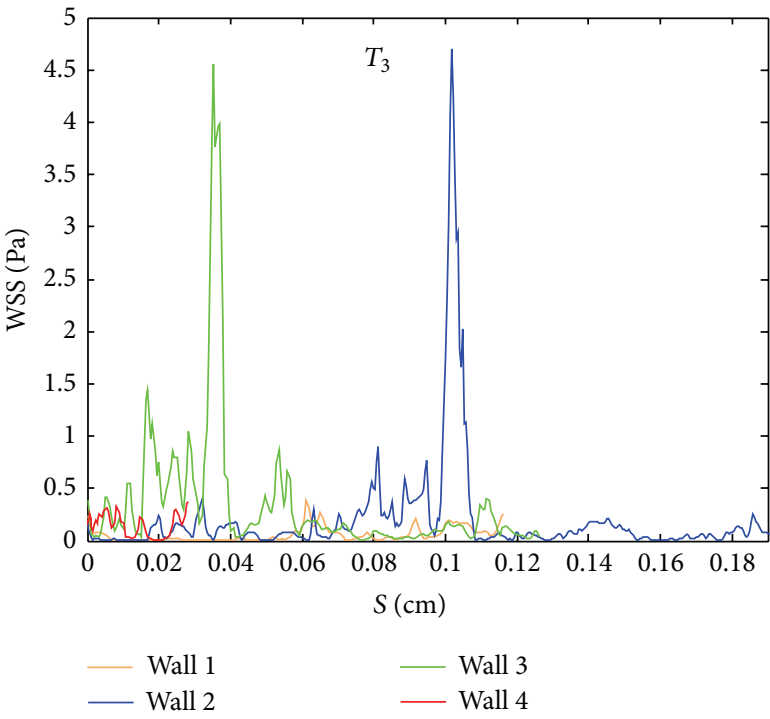

(d)

FIGURE 5: (a) Boundary plots of coronary arterial walls 1, 2, 3, and 4 shown in red, green, blue, and brown, respectively. (b)-(d) WSS distribution along coronary walls at time $T_{1}, T_{2}$, and $T_{3}$, respectively.

out during the first three cycles. The oscillatory shear index for the pulsatile flow simulation is calculated as [4]

$$
\text { OSI }=0.5\left[1-\frac{\left|\int_{0}^{T} \vec{\tau}_{w} d t\right|}{\int_{0}^{T}\left|\vec{\tau}_{w}\right| d t}\right] .
$$

\section{Results and Discussion}

3.1. Wall Shear Stress. The wall shear stress is among the first mechanisms proposed to relate the blood flow to the localization of atherosclerosis [18] and the plaque rupture [19, 20]. Figures $2-5$ demonstrate the WSS distributions along the coronary walls versus the curve length $(S)$, at three different time instants, for four different diseased coronary arteries. In the present study, the WSS is defined as the magnitude of all three components at any location on the wall.

Figure 2(a) demonstrates the boundary plots for the images of the first stenosed coronary artery (Figure 1(a)). The WSS distributions along the coronary walls versus curve length at diastole, the peak systole, and accelerating phase are shown in Figures 2(b)-2(d), respectively. The areas with maximum WSS can be observed along the distal side and on the throat of the stenosis at diastole time point. Note that a minimum value of WSS appears at the top of the atherosclerotic plaque at the peak systole and accelerating 


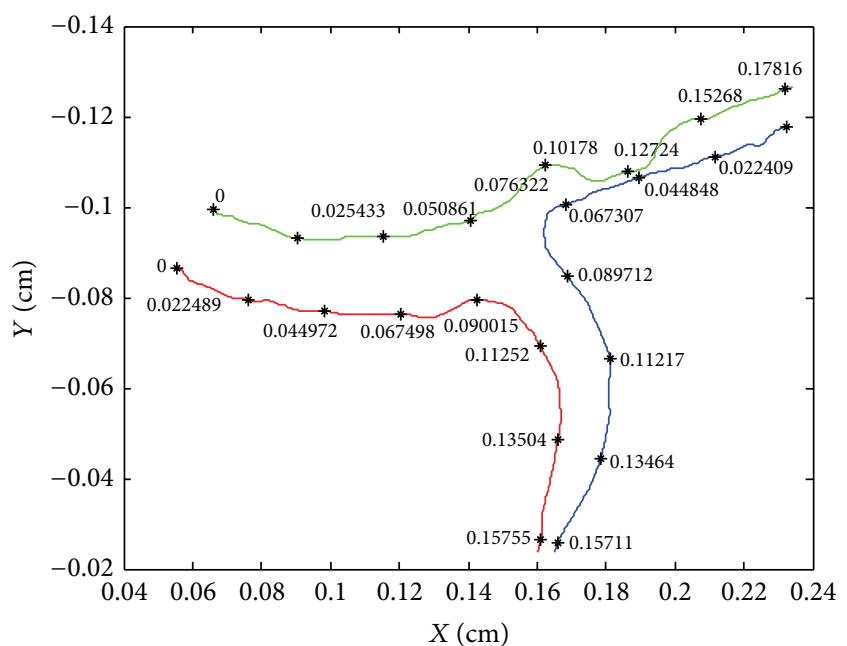

(a)

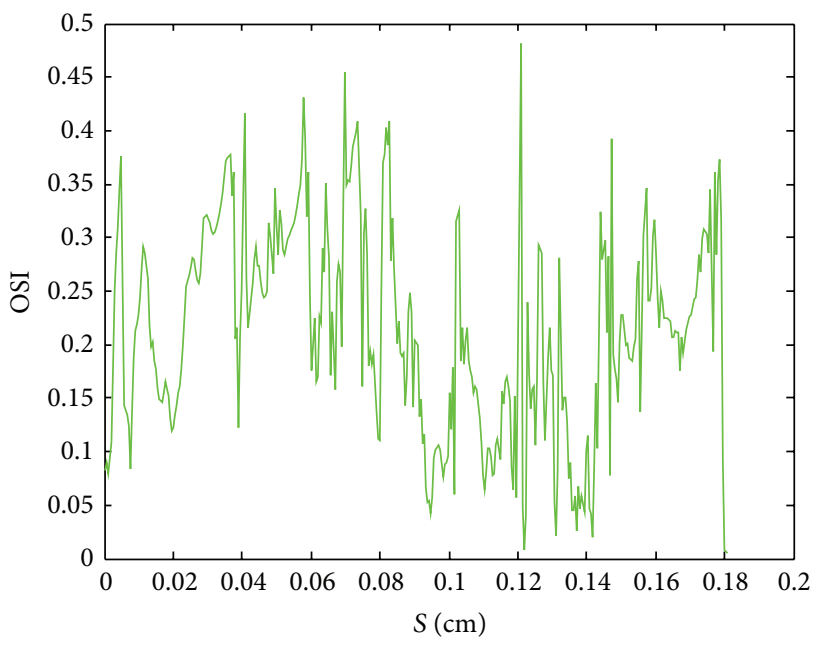

(c)

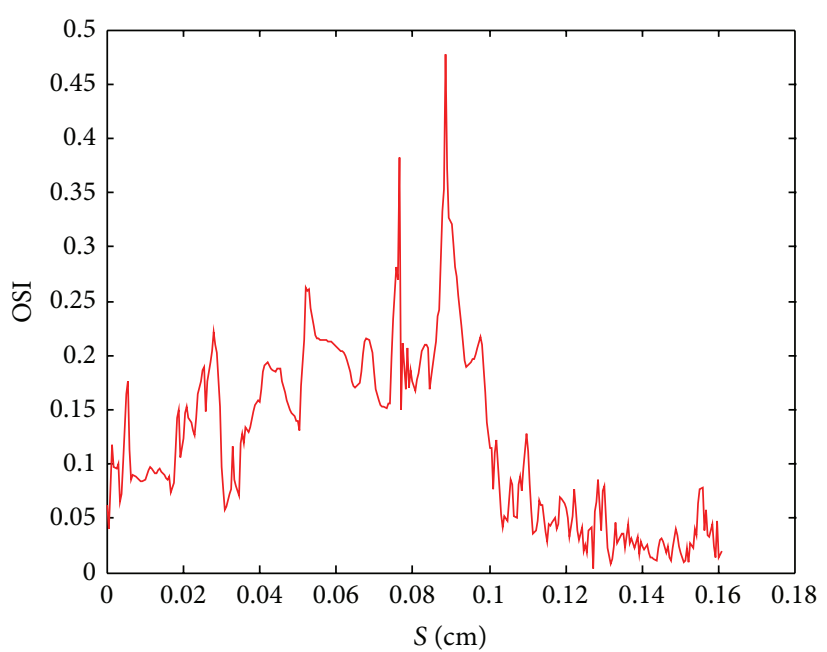

(b)

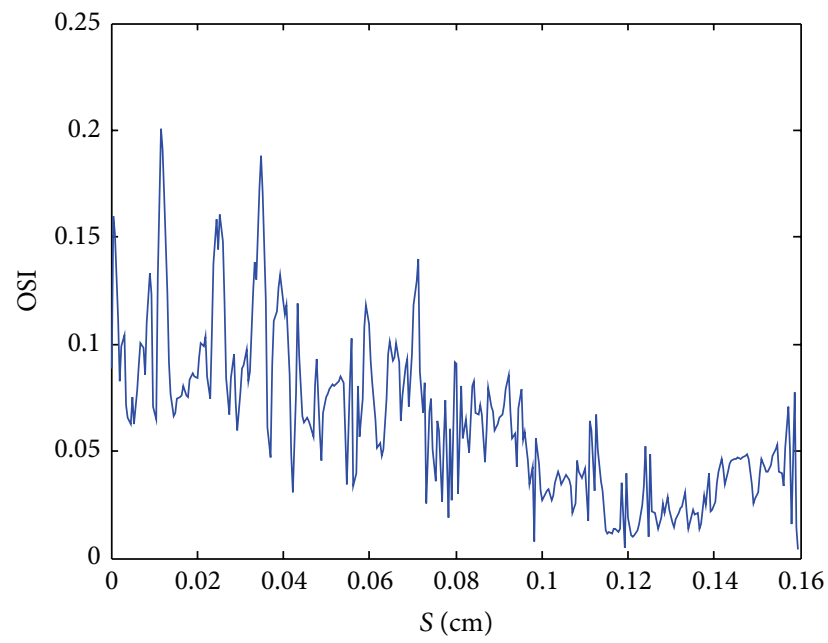

(d)

FIGURE 6: (a) Boundary plots of the coronary arterial walls 1, 2 and 3 shown in red, green, and blue, respectively. (b)-(d) OSI distribution along coronary walls 1,2 , and 3 , respectively.

time points. It is worth mentioning that the surfaces of the coronary walls, in the present study, are generated based on the original images of patients. Surfaces are not smoothed after being read in MATLAB. The reason is associated with the aim of the current study at the investigation of the connection of atherosclerotic plaque to the local characteristics of the luminal surface. As Figure 2(a) shows, the wall on the top of the plaque is not smooth which is according to the original histological image (Figure 1(a)). This is the region with a minimum magnitude of WSS.

Figures 3(a)-3(d) show the WSS versus curve length along the walls of the second stenosed coronary artery (Figure 1(b)). The maximum magnitude of WSS occurs at the top of the plaque. Another stenosed region shows the second peak of WSS values at systole and accelerating time points.

Figures 4(a)-4(d) demonstrate the WSS along the walls of the third diseased coronary artery (Figure 1(c)), while Figures 5(a)-5(d) demonstrate the WSS along the walls of the forth diseased coronary artery (Figure $1(\mathrm{~d})$ ). Maximum of the WSS magnitude occurs at the throat of the stenoses in both models, at diastole time point. As seen in Figures 4(c) and 4(d), another maximum value of WSS is observed at systole and accelerating time points in the region with high curvature. In Figures 5(c) and 5(d), the proximal side of the plaque shows relatively high magnitude of WSS at systole and accelerating time points. In this artery the stenosis has been created at the bifurcation.

3.2. Oscillatory Shear Index (OSI). OSI is a known predictor of formation of atherosclerosis and vulnerability for plaque in coronary arteries. Figures 6(b)-6(d) demonstrates OSI for the first stenosed coronary artery. Figure 6(b) shows that OSI has high values at the high curvature region. Therefore, this location may be predicted to experience lesion in the future. As shown in Figure 6(c), OSI is high along the stenosed wall of coronary artery (shown in green in Figure 6(a)). OSI 


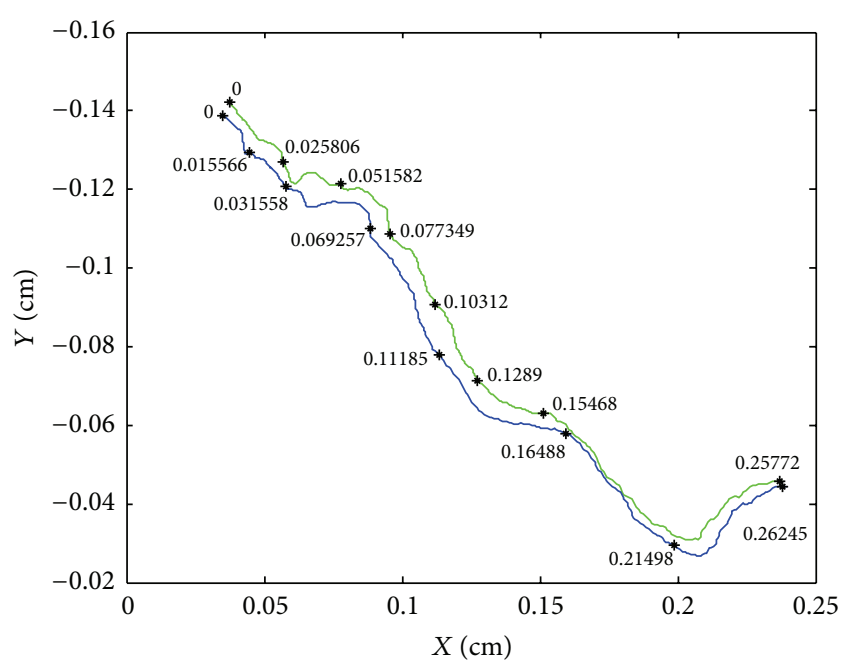

(a)

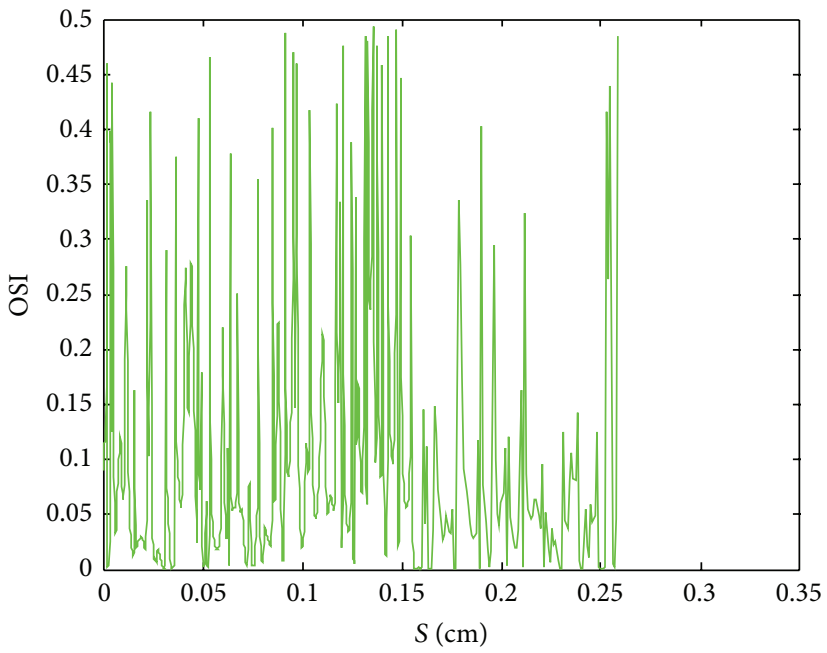

(b)

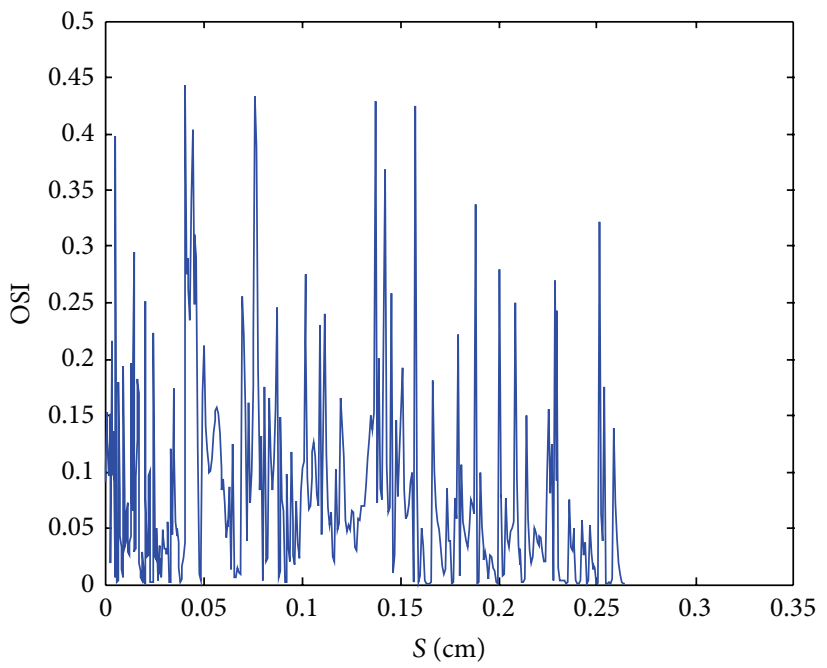

(c)

FIGURE 7: (a) Boundary plots of the coronary arterial walls 1 and 2 shown in green and blue, respectively. (b)-(c) OSI distribution along coronary walls 1 and 2 .

distribution reveals that the whole length of the green wall is vulnerable to atherosclerosis. Figure 6(d) demonstrates a relatively high OSI values at the curvature region along the wall.

Figures 7, 8, and 9 show OSI for the next stenosed coronary arteries. Maximum values of OSI occur in stenosed regions, in all arteries. The next area with maximum of OSI is the region with high curvature. Note that this behavior is observed even in healthy curves.

\section{Conclusions}

In this study, pulsatile blood flow is studied in four reconstructed models of the diseased coronary arteries from histological images. Surface smoothing and approximation of the vessels with regular shapes such as straight or curved cylinders are avoided unlike most of earlier studies. The real geometric model and the conditions of pulsatile flow allow to spot vulnerable areas to the development of atherosclerotic lesions. The results indicate a strong dependence of the hemodynamics on the morphology of atherosclerotic lesion. Distinctive flow patterns appear in different stenosed regions corresponding to the specific geometry of the arteries. The OSI is a well-known predictor of formation of atherosclerosis and vulnerability for plaque in coronary arteries. High OSI has been widely used as indicators of atherosclerosis because the region with high OSI is likely to experience stagnation or backflow. This is strongly related to the progression of atherosclerosis because it affects the arrangement of endothelial cells in adjacent tissues. It has been shown that the areas with high values of OSI are usually located in the regions where wall shear stress is low. In the present study, OSI is high in diseased region as well as healthy regions with low values of WSS which can be predicted to experience 


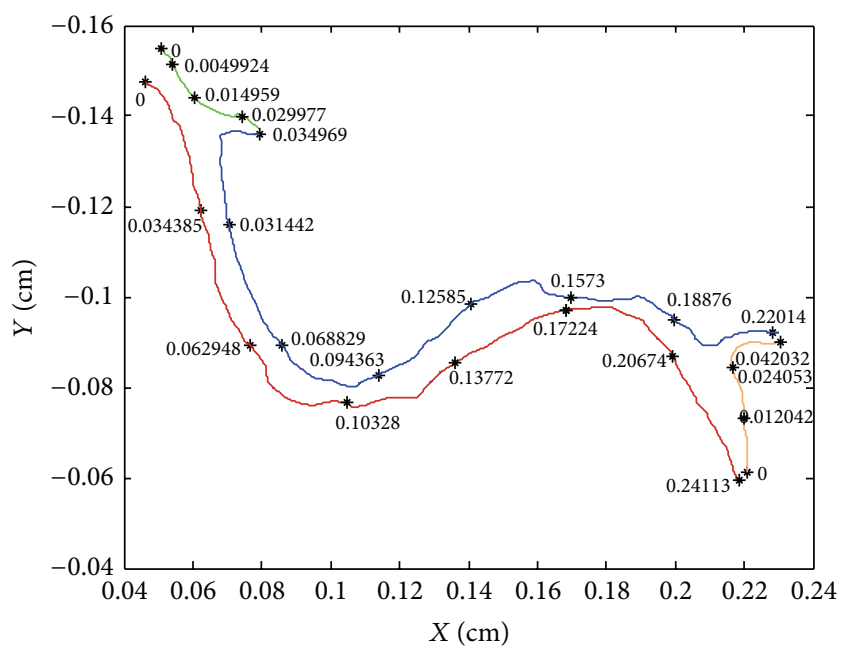

(a)

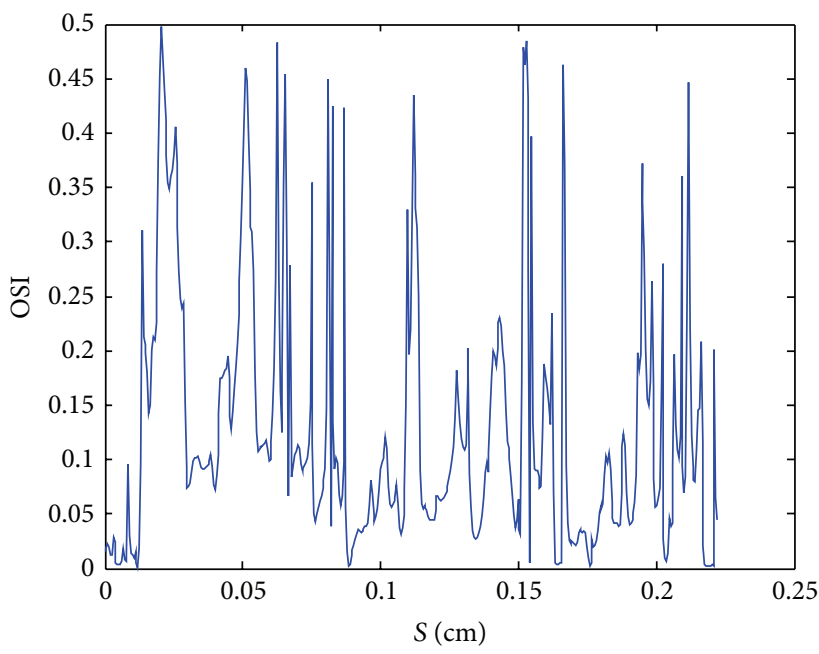

(c)

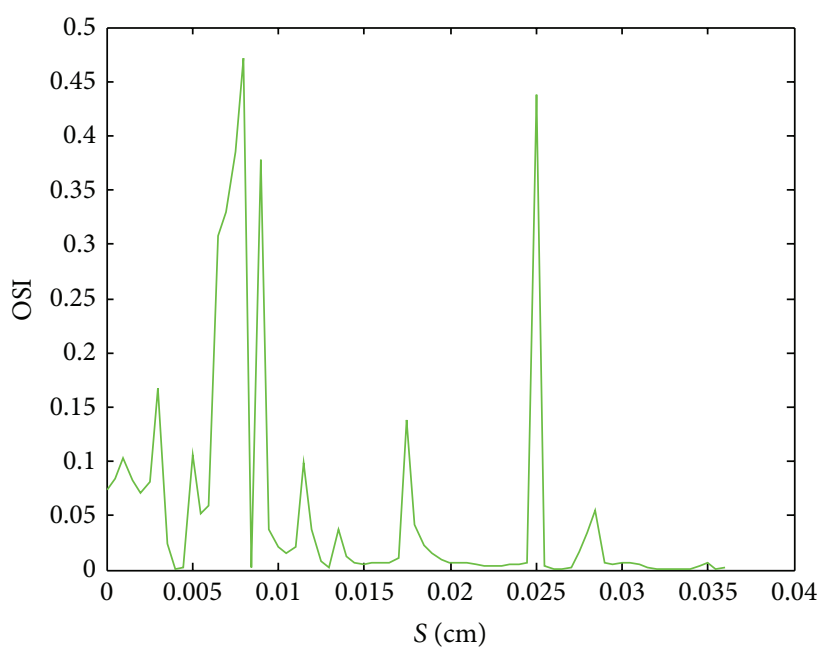

(b)

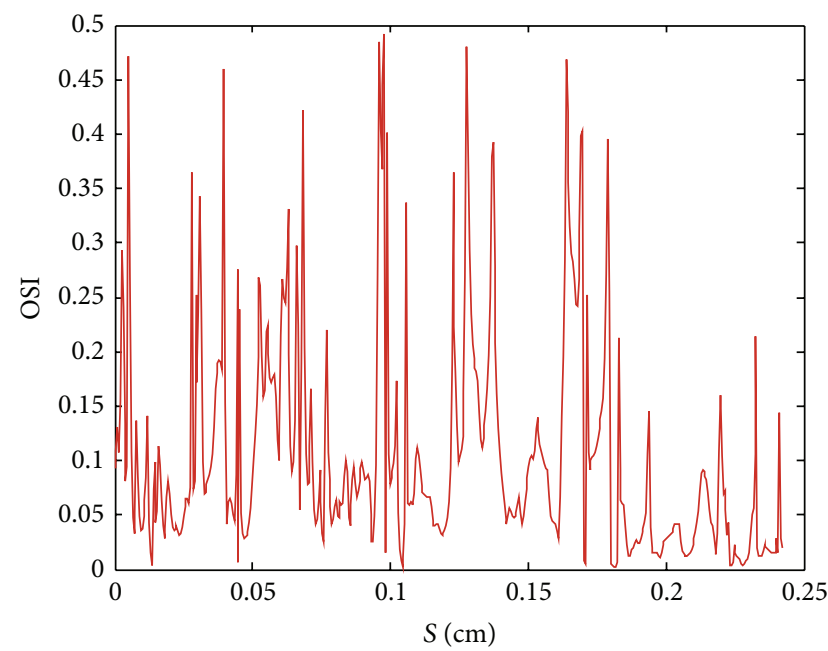

(d)

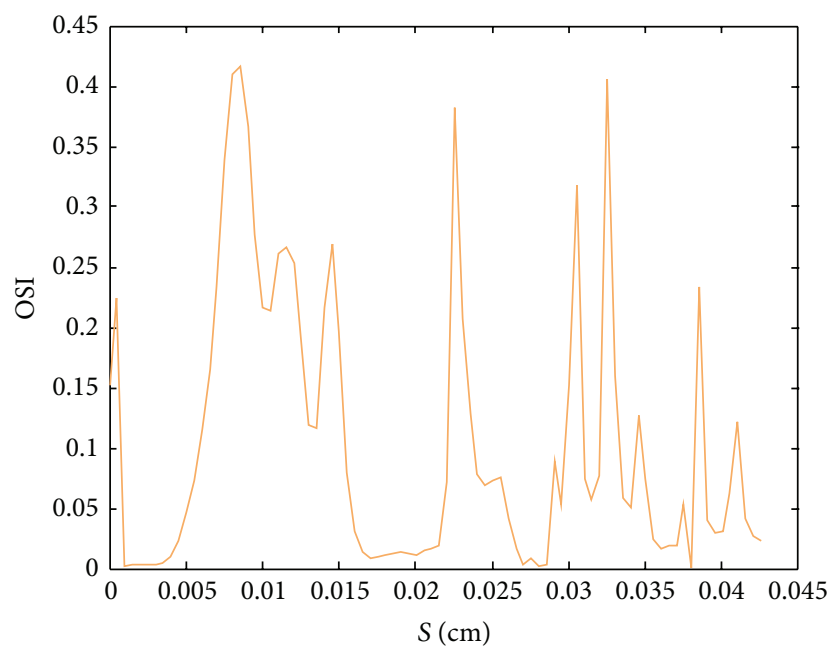

(e)

FIGURE 8: (a) Boundary plots of coronary arterial walls 1, 2, 3, and 4 shown in red, green, blue, and brown, respectively. (b)-(e) OSI distribution along coronary walls $1,2,3$, and 4 . 

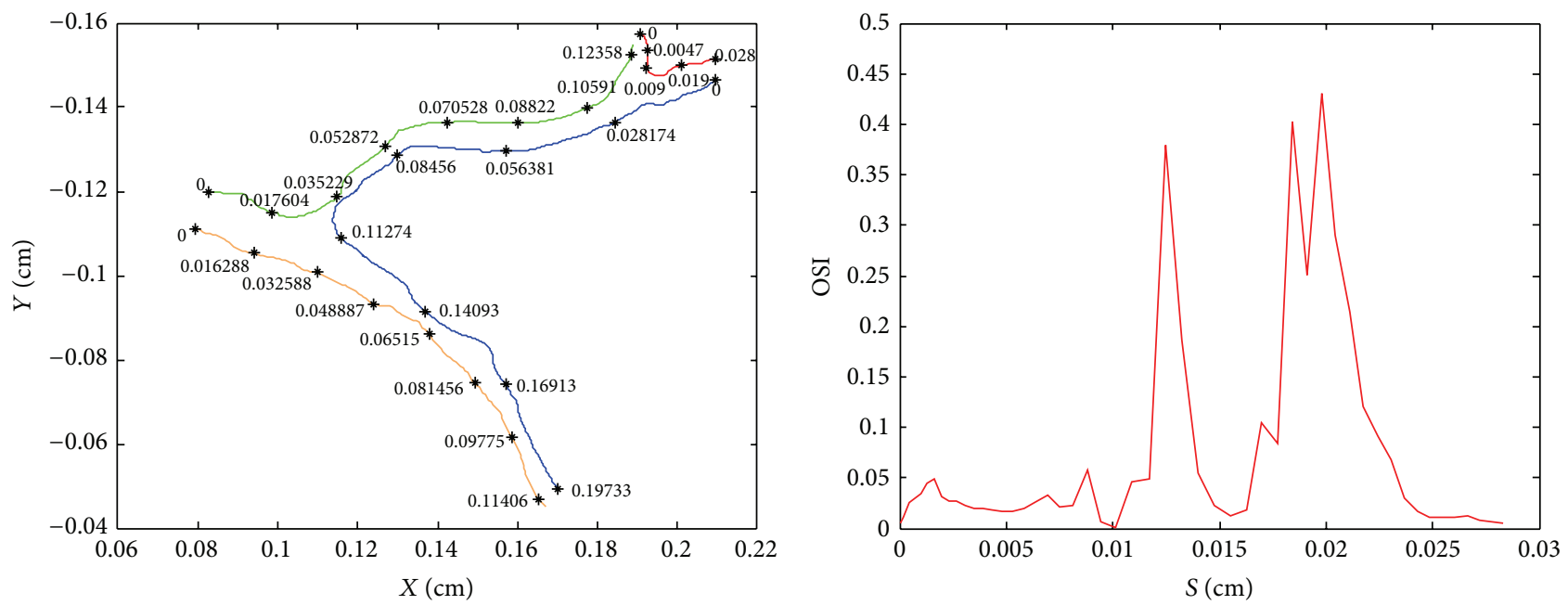

(a)

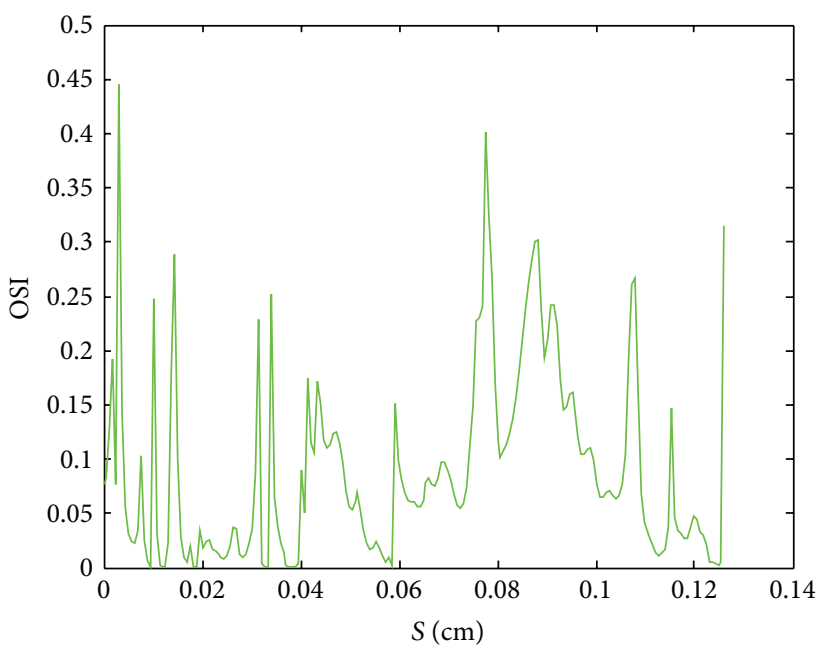

(b)

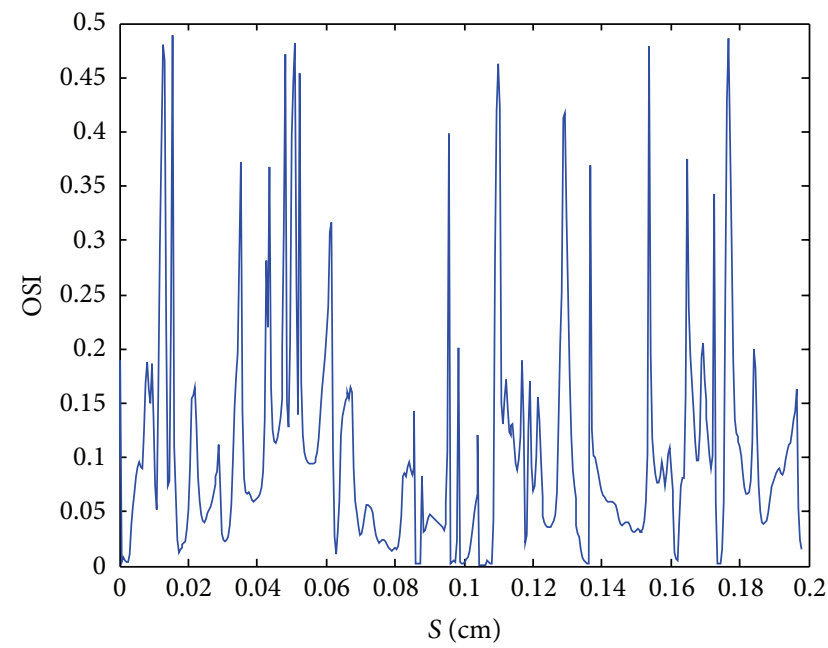

(c)

(d)

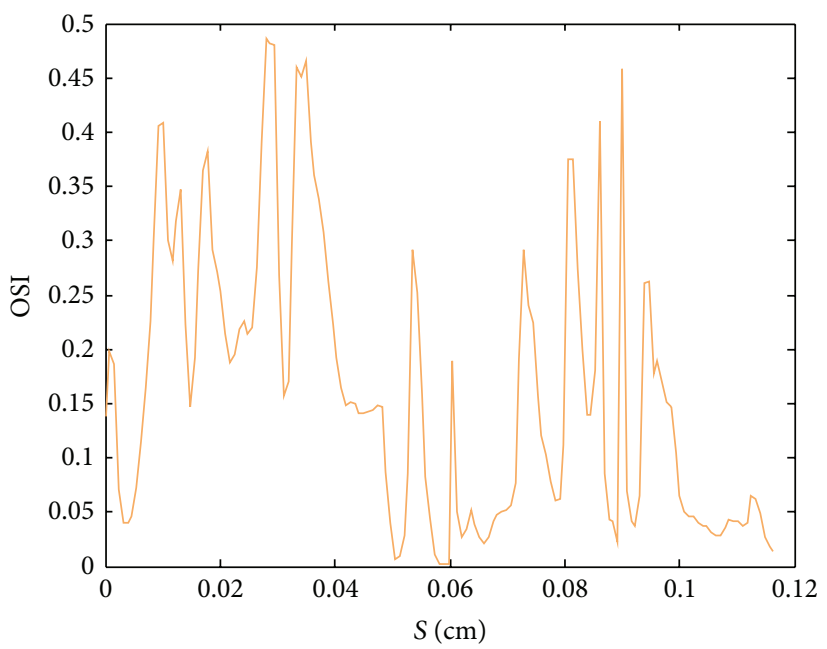

(e)

FIGURE 9: (a) Boundary plots of coronary arterial walls 1, 2, 3, and 4 shown in red, green, blue, and brown, respectively. (b)-(e) OSI distribution along coronary walls $1,2,3$, and 4 . 
lesion in the future. The findings support the low shear stress and oscillatory shear stress theory which relates abnormal biological effects inside arteries to high OSI. Moreover, the present study demonstrates the shear environment in longitudinal section of the diseased coronary. The stenosis affects the WSS locally along the diseased arterial wall as well as adjacent walls. The maximum value of WSS is observed in the throat of stenosis which highlights the risk of plaque rupture. The arterial plaque can break apart as a result of high stresses acting on or within the arterial wall. The severity and the peripheral distribution of stenosis are the crucial geometric factors influencing the risk of breakage.

It is hoped that computational studies of this type will enable scientists to directly relate the hemodynamics to the formation and progression of atherosclerosis in the coronary arteries. More than $60 \%$ of myocardial infarction is caused by the rupture of vulnerable plaques [17]. A significant contribution of the present study is the demonstration of robust and highly efficient computational methods for studying the pulsatile blood flow field in different stenosed coronary arteries. The current study is the first detailed numerical investigation of the flow field within the diseased coronary arteries which are reconstructed from histological images. The spatial and temporal variation of wall shear stress from the present study can be fed into other models to investigate the effect of wall shear stress on stresses and strains that individual endothelial cells may tolerate locally.

\section{Conflict of Interests}

None of the authors has a conflict of interests regarding this work.

\section{Acknowledgments}

M. Dabagh and P. Jalali would like to thank the financial support from the Faculty of Technology in Lappeenranta University of Technology and Tukisäätiö Foundation. Authors kindly acknowledge the Pathology Core Lab in the Winship Cancer Institute, Emory University, for preparing the images.

\section{References}

[1] M. Dabagh, P. Jalali, and J. M. Tarbell, “The transport of LDL across the deformable arterial wall: the effect of endothelial cell turnover and intimal deformation under hypertension," American Journal of Physiology-Heart and Circulatory Physiology, vol. 297, no. 3, pp. H983-H996, 2009.

[2] J. M. Tarbell, "Mass transport in arteries and the localization of atherosclerosis," Annual Review of Biomedical Engineering, vol. 5, pp. 79-118, 2003.

[3] N. Shahcheraghi, H. A. Dwyer, A. Y. Cheer, A. I. Barakat, and T. Rutaganira, "Unsteady and three-dimensional simulation of blood flow in the human aortic arch," Journal of Biomechanical Engineering, vol. 124, no. 4, pp. 378-387, 2002.

[4] D. Mori, T. Hayasaka, and T. Yamaguchi, "Modeling of the human aortic arch with its major branches for computational fluid dynamics simulation of the blood flow," JSME International Journal, vol. 45, no. 4, pp. 997-1002, 2002.
[5] L. Morris, P. Delassus, A. Callanan et al., "3-D numerical simulation of blood flow through models of the human aorta," Journal of Biomechanical Engineering, vol. 127, no. 5, pp. 767775, 2005.

[6] C.-Y. Wen, A.-S. Yang, L.-Y. Tseng, and J.-W. Chai, "Investigation of pulsatile flowfield in healthy thoracic aorta models," Annals of Biomedical Engineering, vol. 38, no. 2, pp. 391-402, 2010.

[7] H. S. Ryou, S. Kim, S. W. Kim, and S. W. Cho, "Construction of healthy arteries using computed tomography and virtual histology intravascular ultrasound," Journal of Biomechanics, vol. 45, pp. 1612-1618, 2012.

[8] P. Vasava, P. Jalali, M. Dabagh, and P. J. Kolari, "Finite element modelling of pulsatile blood flow in idealized model of human aortic arch: study of hypotension and hypertension," Computational and Mathematical Methods in Medicine, vol. 2012, Article ID 861837, 14 pages, 2012.

[9] J.-M. Zhang, L. P. Chua, D. N. Ghista, S. C. M. Yu, and Y. S. Tan, "Numerical investigation and identification of susceptible sites of atherosclerotic lesion formation in a complete coronary artery bypass model," Medical and Biological Engineering and Computing, vol. 46, no. 7, pp. 689-699, 2008.

[10] Y. Huo, T. Wischgol, and G. S. Kassab, "Flow patterns in threedimensional porcine epicardial coronary arterial tree," American Journal of Physiology-Heart and Circulatory Physiology, vol. 293, no. 5, pp. H2959-H2970, 2007.

[11] A. M. Malek, S. L. Alper, and S. Izumo, "Hemodynamic shear stress and its role in atherosclerosis," Journal of the American Medical Association, vol. 282, no. 21, pp. 2035-2042, 1999.

[12] M. Khakpour and K. Vafai, "Critical assessment of arterial transport models," International Journal of Heat and Mass Transfer, vol. 51, no. 3-4, pp. 807-822, 2008.

[13] Ö. Smedby, "Do plaques grow upstream or downstream?: an angiographic study in the femoral artery," Arteriosclerosis, Thrombosis, and Vascular Biology, vol. 17, no. 5, pp. 912-918, 1997.

[14] A. Feintuch, P. Ruengsakulrach, A. Lin et al., "Hemodynamics in the mouse aortic arch as assessed by MRI, ultrasound, and numerical modeling," American Journal of Physiology-Heart and Circulatory Physiology, vol. 292, no. 2, pp. H884-H892, 2007.

[15] K. Jozwik and D. Obidowski, "Numerical simulations of the blood flow through vertebral arteries," Journal of Biomechanics, vol. 43, no. 2, pp. 177-185, 2010.

[16] T. Kim, A. Y. Cheer, and H. A. Dwyer, "A simulated dye method for flow visualization with a computational model for blood flow," Journal of Biomechanics, vol. 37, no. 8, pp. 1125-1136, 2004.

[17] J. T. Dodge Jr., B. G. Brown, E. L. Bolson, and H. T. Dodge, "Lumen diameter of normal human coronary arteries: influence of age, sex, anatomic variation, and left ventricular hypertrophy or dilation," Circulation, vol. 86, no. 1, pp. 232-246, 1992.

[18] R. Torii, N. B. Wood, A. D. Hughes et al., "A computational study on the influence of catheter-delivered intravascular probes on blood flow in a coronary artery model," Journal of Biomechanics, vol. 40, no. 11, pp. 2501-2509, 2007.

[19] J. K. Li, Dynamic of the Vascular System, World Scientific Publishing, Hackensack, NJ, USA, 2004.

[20] R. M. Nerem, W. A. Seed, and N. B. Wood, "An experimental study of the velocity distribution and transition to turbulence in the aorta," Journal of Fluid Mechanics, vol. 52, no. 1, pp. 14$1972,1972$. 


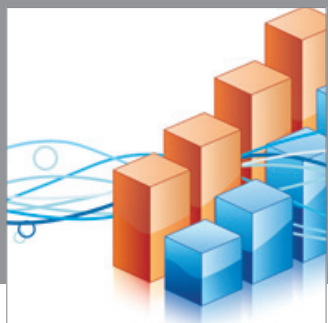

Advances in

Operations Research

mansans

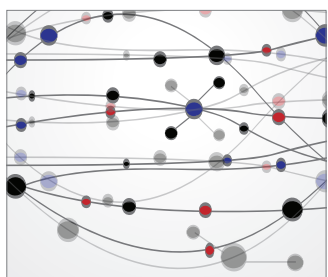

The Scientific World Journal
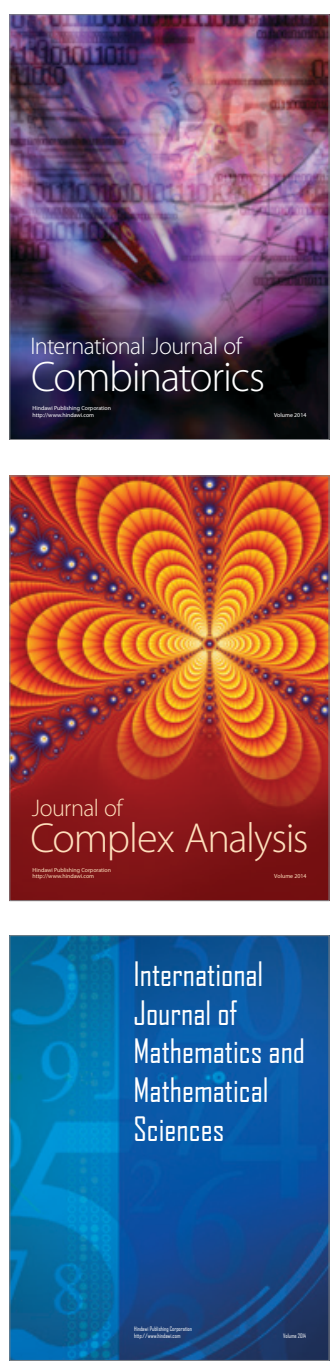
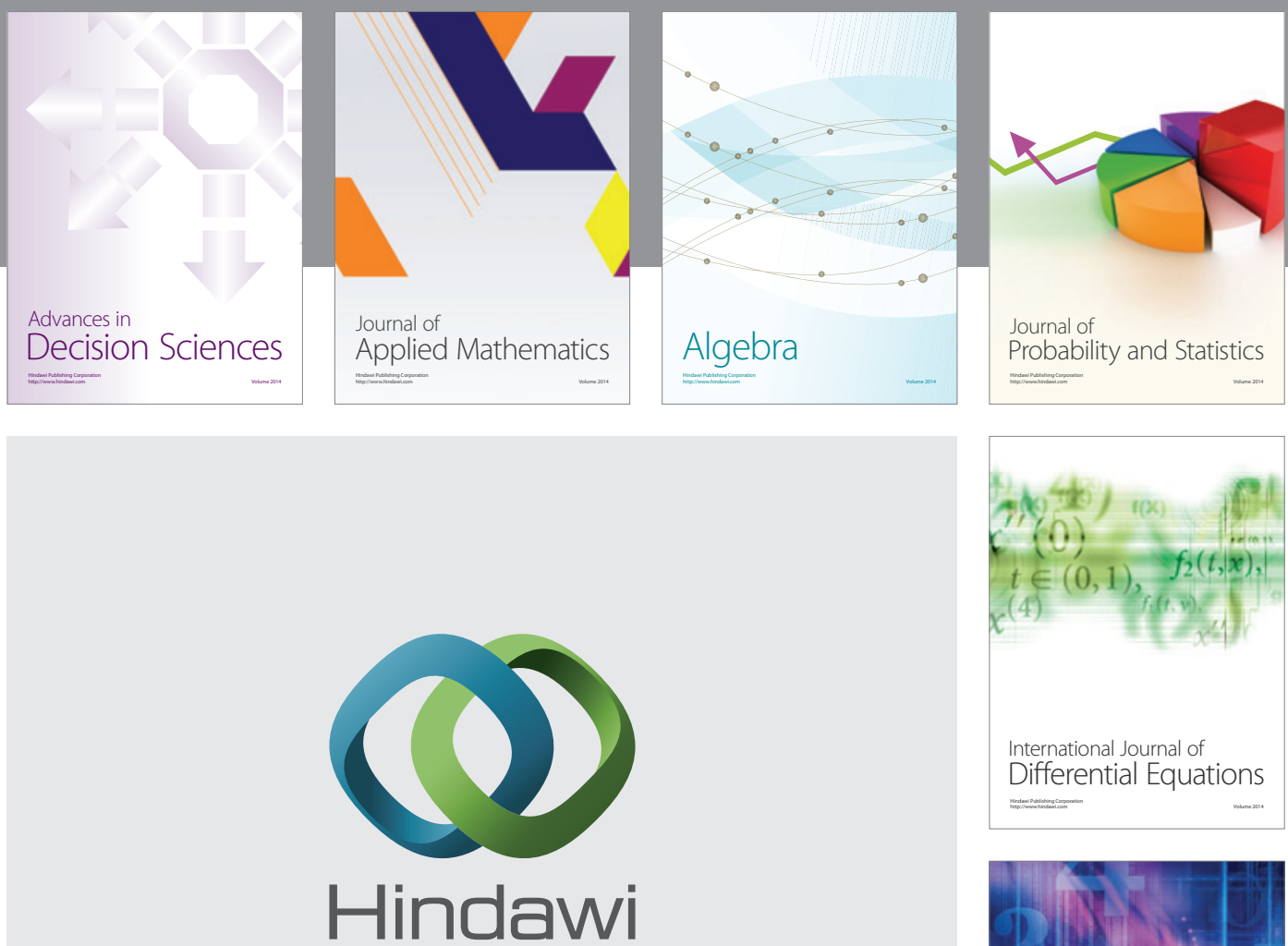

Submit your manuscripts at http://www.hindawi.com
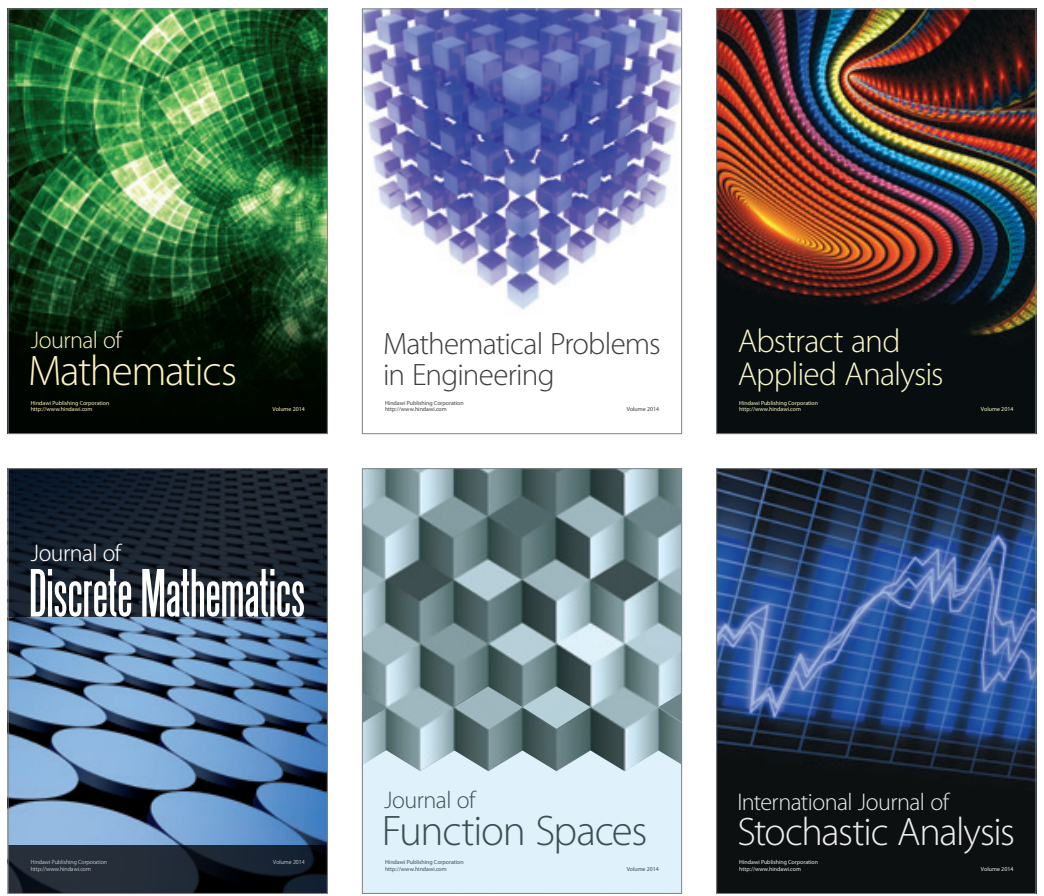

Journal of

Function Spaces

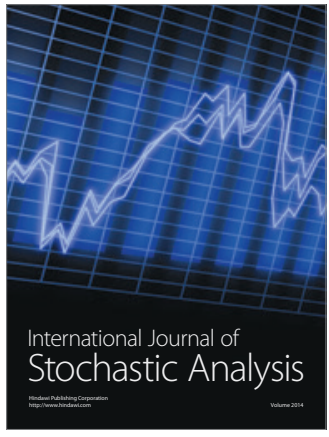

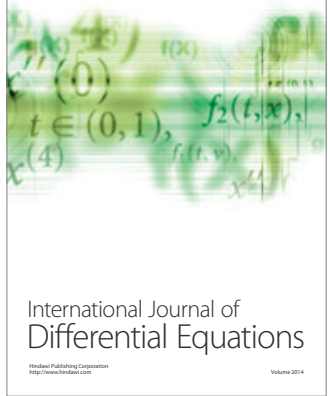
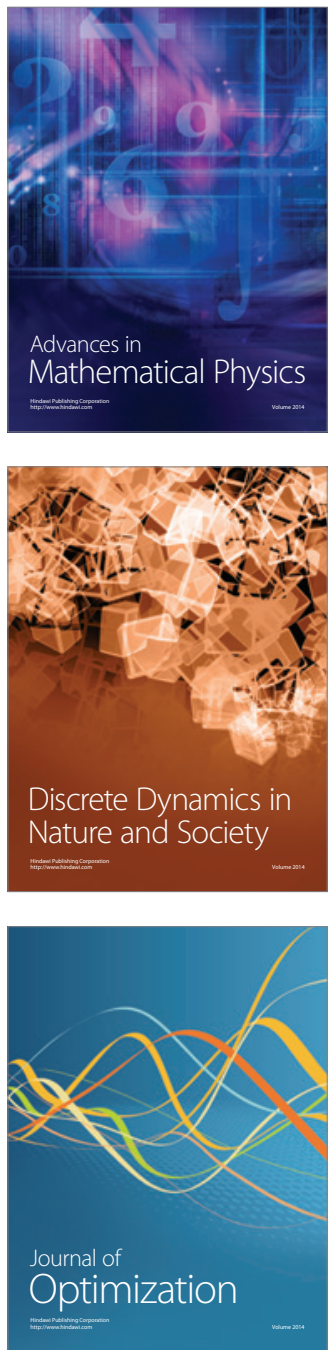